\title{
Molecular analysis of three patients with interstitial deletions of chromosome band $14 \mathrm{q} 31$
}

Barbara C Byth, M Teresa Costa, Ikuko E Teshima, William G Wilson, Nigel P Carter, Diane W Cox
$(\mathrm{CA})_{\mathrm{n}}$ repeats, localised to the distal portion of chromosome 14, to define at the molecular level the extent of the interstitial 14q31 deletions in three patients (HSC 1251, 1141, and 1337).

Analysis of the $14 \mathrm{q} 31$ deletions in our patients refines the localisation of a number of markers within the region. The gene responsible for Machado-Joseph disease (MJD) has been localised to this chromosome region by linkage analysis in Japanese ${ }^{67}$ and Azorean families. ${ }^{8}$ MJD is a multisystem neurodegenerative disease inherited as an autosomal dominant trait. The gene, recently cloned, contains a trinucleotide repeat, which is expanded in MJD. The gene lies close to or within the deleted region in two of the patients.

\section{Materials and methods}

PATIENTS

Patient 1 (HSC 1251)

This female patient has been reported previously as having a cytogenetically detectable deletion of all of chromosome band q31 (deletion of $14 q 24 \rightarrow 14 q 32.1) .^{2}$ She had some of the characteristic facial features seen in other cases with interstitial deletions of chromosome 14: bushy eyebrows, downward slanted palpebral fissures, epicanthic folds, protuberant ears, an upturned nose with wide nasal bridge, micrognathia, and dental abnormalities. She also has mild psychomotor retardation. Both parents had normal karyotypes and were available for study.

A characteristic constellation of features found in patients with interstitial deletions of the distal portion of chromosome 14 $(14 q 23 \rightarrow q 32)$. Ten such cases have been described previously. ${ }^{1-4} \mathrm{~A}$ characteristic facies (including bushy eyebrows, epicanthic folds, strabismus, small nose with flat nasal bridge, microcephaly, low set and deformed ears, carp mouth, dental abnormalities, narrow and arched palate, micrognathia) is often observed. In addition, single palmar creases, heart defects, skeletal abnormalities, and genitourinary abnormalities including cryptorchidism have been reported in some cases. The extent of the chromosomal deletions in these patients is probably quite heterogeneous, so to date phenotype-genotype correlations have been impossible. Some of the same features, particularly a similar facial appearance, are seen in some patients reported to have terminal deletions of chromosome 14 (deletion of $14 \mathrm{q} 32.3 \rightarrow$ qter) which do not overlap with the deletions of band $14 \mathrm{q} 31 .{ }^{5}$ We have used simple sequence repeat polymorphisms, principally

\section{Patient 2 (HSC 1141)}

This female patient was initially reported as having a terminal deletion of chromosome 14 (deletion of $14 \mathrm{q} 31.1 \rightarrow \mathrm{qter}) .{ }^{4}$ She has not been located for repeat karyotype analysis. She had microcephaly, protuberant ears, epicanthic folds, micrognathia, gingival hypertrophy, and a narrow palate. Her motor milestones were normal, but she was diagnosed as having mild parents had normal karyotypes.

\section{Patient 3 (HSC 1337)}

This patient and his family have not been reported elsewhere. The proband was referred for assessment of developmental delay. He was the product of a normal pregnancy and delivery; birth weight was $4600 \mathrm{~g}$. Early gross motor milestones were normal, but at the age of 5 developmental delay at 5 years of age. Both
Received 27 September 1994

Revised version accepted for publication

27 February 1995 


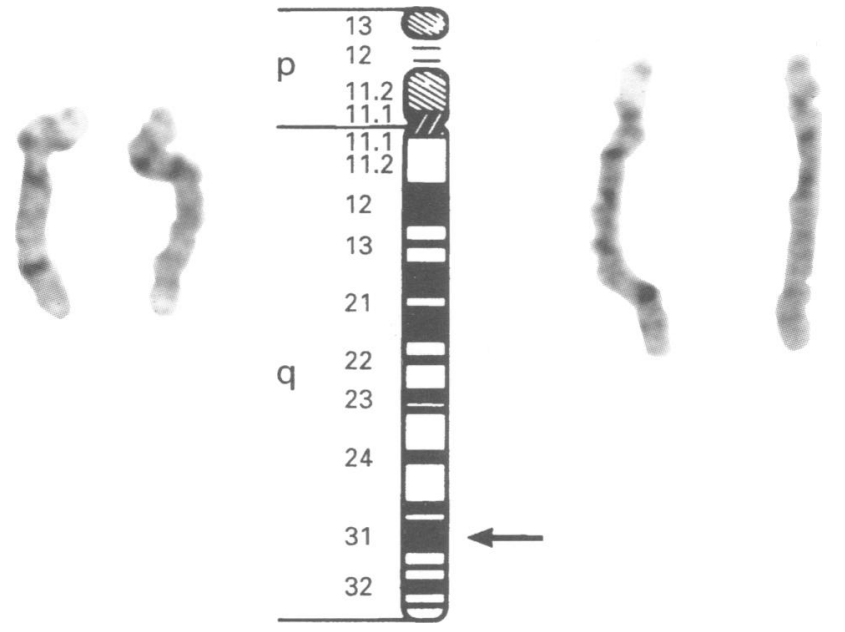

Figure 1 Ideogram of chromosome 14 with a pair of chromosomes 14 and del(14) from patient 3 on either side; in each pair the normal 14 is to the left and the del(14) is to the right. The arrow indicates the band that is partially deleted.

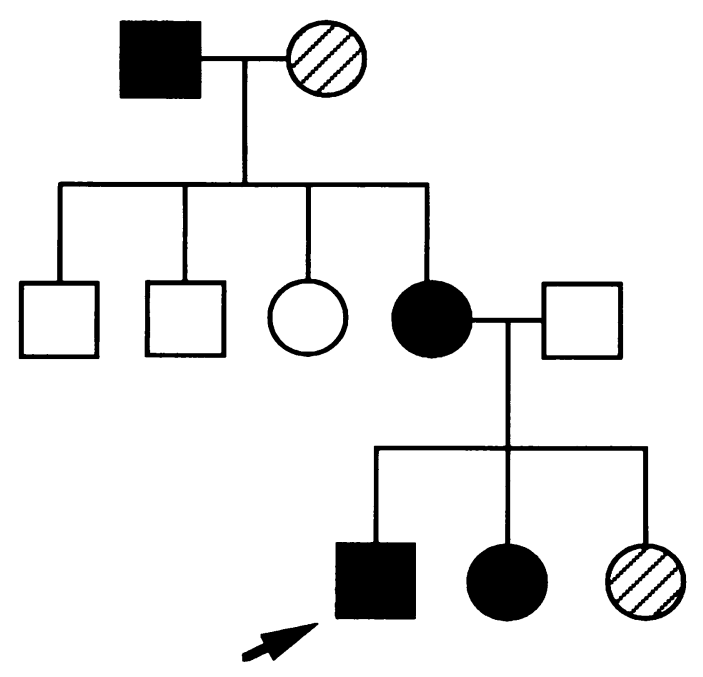

Figure 2 Pedigree of patient 3 (indicated by the arrow). Filled symbols indicate the presence of the deletion. Shaded symbols indicate subjects tested and shown to be of normal karyotype.

years he was lagging in all areas especially in speech and language skills. Physical examination was normal except for cupped ears. Chromosome analysis showed an interstitial deletion of part of chromosome band $14 \mathrm{q} 31$

Results of molecular analysis of deletion patients

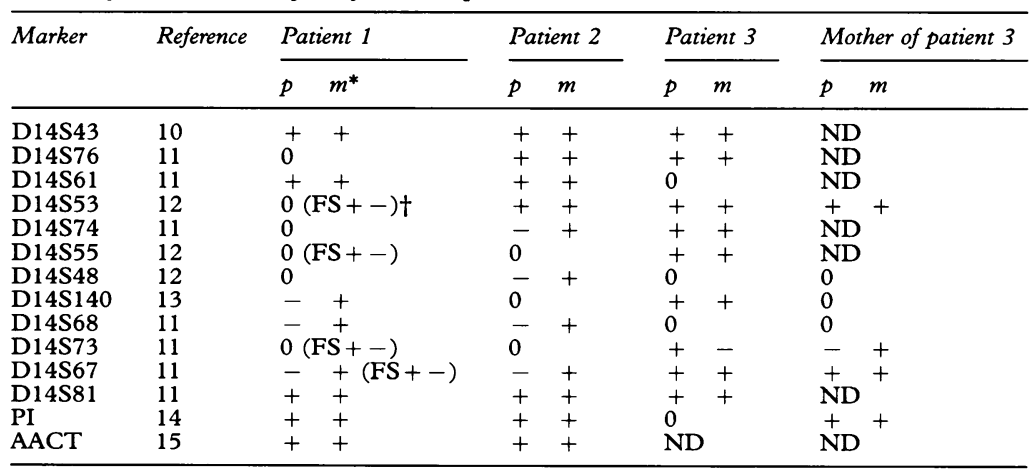

$* \mathrm{p}=$ paternal allele; $\mathrm{m}=$ maternal allele; + denotes presence and - absence of allele; 0 denote an uninformative result; $\mathrm{ND}=$ typing not done.

tFS indicates the use of PCR analysis of flow sorted chromosomes for patient 1. Results are given first for the normal chromosome, second for the deleted chromosome. (fig 1). The same abnormality was found in a younger sister with a similar phenotype, and in his mother and maternal grandfather (fig 2). The mother had delayed speech development and learning difficulties; she functions in the borderline normal IQ range. Her three sibs are intellectually normal, two having completed university education. Her physical examination was normal except for cupped ears. The grandfather completed elementary school and worked successfully as a tradesman until retirement. He had cupped ears but no other dysmorphic features. DNA samples were obtained from both parents and from the maternal grandparents.

\section{ANALYSIS OF SIMPLE SEQUENCE REPEAT} POLYMORPHISMS

Fourteen markers localised to the distal portion of chromosome 14 were used in the analysis (table). These markers are polymorphic $(\mathrm{CA})_{\mathrm{n}}$ repeats, with the exceptions of the AACT marker, which is a complex dinucleotide repeat $(\mathrm{TA})_{\mathrm{n}}(\mathrm{GA})_{\mathrm{n}}{ }^{15}$ and of D14S140 which is a tetranucleotide repeat. ${ }^{13}$ References to primer sequences are given in the table. Genomic DNA (10 ng) was amplified in $10 \mu \mathrm{l}$ PCR reactions containing the following reagents: $50 \mathrm{mmol} / 1 \mathrm{KCl}, 10 \mathrm{mmol} / 1$ Tris-Cl $\mathrm{pH} 8 \cdot 4,1$ or $2 \mathrm{mmol} / 1 \mathrm{MgCl}_{2} ; 200 \mu \mathrm{mol} / 1 \mathrm{dCTP}, \mathrm{dGTP}$, dTTP, $25 \mu \mathrm{mol} / 1$ dATP; $2 \mu \mathrm{Ci} \alpha-\left[{ }^{35} \mathrm{~S}\right]-\mathrm{dATP}$; $0.5 \mu \mathrm{mol} / 1$ each primer and $0.5 \mathrm{U}$ Amplitaq (Perkin Elmer). The amplification conditions were 30 cycles of: denaturing $94^{\circ} \mathrm{C} / 30$ seconds, annealing 30 seconds, extension $72^{\circ} \mathrm{C} / 30$ seconds. The PCR products were resolved on $6 \%$ sequencing gels and visualised by autoradiography. The D14S140 marker was analysed without the inclusion of $\alpha-\left[{ }^{35} S\right]-d A T P$ on $5 \%$ non-denaturing polyacrylamide gels stained with ethidium bromide.

The order of the markers was derived from linkage analysis, ${ }^{116}$ from physical mapping studies in somatic cell hybrids, ${ }^{17}$ and from the study of a person with a balanced translocation in which D14S48 was proximal to D14S68 and D14S73. ${ }^{18}$ The order of markers D14S68, D14S73, and D14S67 used here was derived from analysis of loss of heterozygosity in renal cell tumours. ${ }^{19} \mathrm{D} 14 \mathrm{~S} 140$ was placed by analysis of recombinant subjects in four pedigrees from the CEPH reference collection (CEPH 1332, 28, 35, and 45) (Byth and Cox, unpublished data).

\section{ANALYSIS OF FLOW SORTED CHROMOSOMES} FROM PATIENT 1

Flow sorted chromosomes were obtained for patient 1 . The deleted and normal chromosomes 14 were separated using bivariate flow karyotype analysis in which the chromosomes were stained with both chromomycin A3 and Hoeschst 33258. The chromosomes were sorted directly into PCR tubes containing $30 \mu \mathrm{l}$ of sterile distilled water by using a dual laser flow cytometer (FACStar Plus, Becton Dickinson) as described previously. ${ }^{20}$ Aliquots of approximately 300 chromosomes were used 

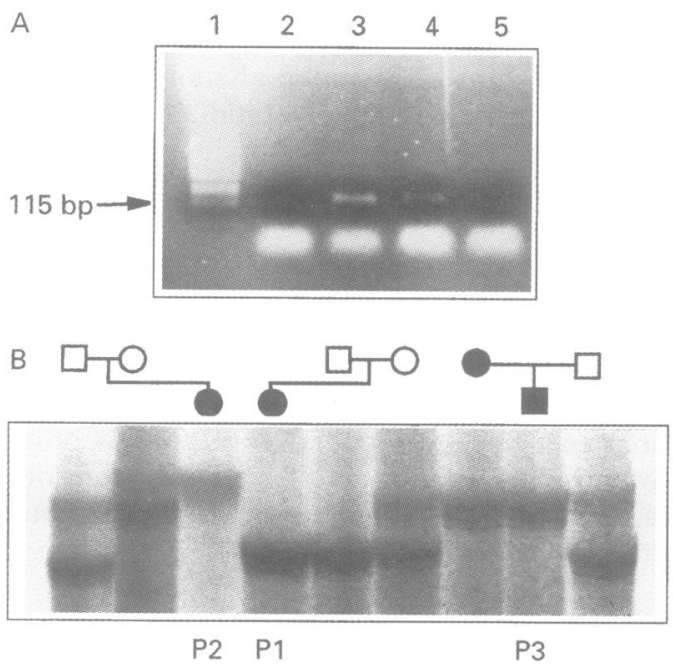

P3

Figure 3 (A) Analysis of flow sorted chromosomes from patient 1 at the D14S67 locus. Lane 1: DNA size marker; lane 2: patient's deleted chromosome 14; lane 3: patient's normal chromosome 14; lane 4: positive control (genomic DNA); lane 5: negative control (no DNA). The size of the amplified product (115 bp) is indicated. This locus is absent from the patient's deleted chromosome 14 and present on her normal chromosome 14 . $(B)$ Results of heterozygosity analysis in the three patients at the D14S48 locus. Patient 2 has a deletion of paternal origin at this locus; patients 1 and 3 are uninformative. (C) Results of heterozygosity analysis of patient 3 , his parents, and maternal grandparents at the D14S73 locus. Patient 3 and his mother have deletions of, respectively, maternal and paternal origin. The major allelic bands are indicated by dots.
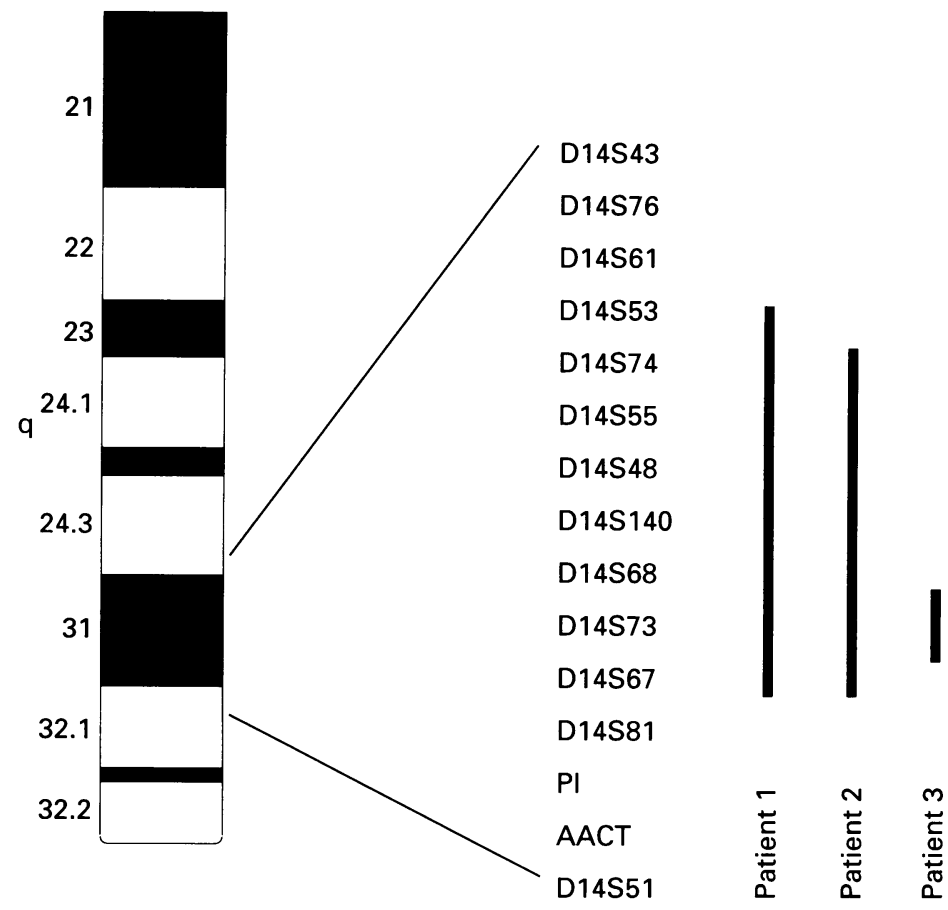

Figure 4 Diagram of the distal portion of chromosome $14 q$ showing the extent of the deletions in the three patients and the possible location of the MFD gene from Sasaki et $a l^{7}$ and St George-Hyslop et al .

in the PCR amplification. The PCR conditions were essentially the same as described above, except that 35 cycles of amplification were performed, the $\alpha-\left[{ }^{35} S\right]$-dATP was omitted, and the concentration of dATP increased to $200 \mu \mathrm{mol} / \mathrm{l}$, and the products were analysed on agarose gels.

\section{Results}

The results of the analyses are given in the table, and examples for three markers, D14S67, D14S48, and D14S73, are shown in fig 3.
Patients are defined as heterozygous $(++)$ or hemizygous ( +-$)$ depending on the inheritance of alleles from their parents. Where the parents share a common allele and the patient has inherited only this allele, it is impossible to determine whether the patient has one or two copies and the result is defined as uninformative $(0)$. The maternal grandparents of patient 3 were also studied.

Patient 1 is deleted at the loci D14S53, D14S55, D14S73, and D14S67, as determined from PCR analysis of the flow sorted chromosomes (fig 3). She is deleted for the paternal allele at D14S140, D14S68, and D14S67, and is uninformative for intervening loci (D14S74 and D14S48). This is consistent with a deletion of paternal origin extending from D14S53 to D14S67. Biochemical analysis showed that her serum concentration of $\alpha_{1}$-antitrypsin was $96 \%$ of normal, whereas that of $\alpha_{1}$-antichymotrypsin was $43 \%$ of normal. The $\alpha_{1}$-antitrypsin (PI) and $\alpha_{1}$-antichymotrypsin (AACT) genes are both localised to chromosome $14 \mathrm{q} 32.1 .^{21}$ The observations led the authors of the initial report to speculate that the AACT locus might be deleted on one of her chromosomes; however, the DNA analysis shows that she is heterozygous at this locus, and so her low serum level of $\alpha_{1}$-antichymotrypsin is unrelated.

Patient 2 was initially described as having a terminal deletion of chromosome 14 . A retrospective examination of her karyotype analysis can alternatively be interpreted as an interstitial deletion. The DNA analysis shows that she is heterozygous, and therefore not deleted, for PI, AACT, and D14S81 and for a number of more telomeric markers (D14S13, D14S16, and D14S20; not shown). ${ }^{22}$ She has a deletion of paternal origin at the D14S74, D14S48 (fig 3B), D14S68, and D14S67 loci, and is uninformative for the intervening markers D14S55, D14S140, and D14S73, consistent with an interstitial deletion extending from D14S74 to D14S67.

Patient 3 and his mother are deleted only at the D14S73 locus (fig 3C); the origin of the

MJD deletion is paternal in the mother and maternal in the patient, consistent with the cytogenetic findings. Both are heterozygous for D14S67, the locus flanking D14S73 on the telomeric side. Although both are uninformative for the locus centromeric to D14S73, D14S68, the grandfather is heterozygous at this locus. As he also carries the deletion, this result suggests that D14S68 does not lie in the deleted region.

The provisional order of markers in the region and the extent of the deletion in each patient is shown in fig 4 . The deletions in patients 1 and 2 provide information on gene order as follows: locus D14S53 lies distal to D14S61 and D14S76, and proximal to D14S74 and D14S55; loci D14S68, D14S73, and D14S67 must lie proximal to D14S81.

\section{Discussion}

There is evidence for a distinct clinical phenotype associated with interstitial deletions of distal chromosome 14 in the $14 \mathrm{q} 31$ region, which includes a characteristic facies, skeletal 
abnormalities, dental abnormalities, and developmental delay. Here we present three patients with interstitial deletions of chromosome $14 \mathrm{q} 31$. One of the three patients, patient 1 , is deleted for all of band $14 \mathrm{q} 31$ (deletion 14q24.3 $\rightarrow 14 \mathrm{q} 32.1$ ) and has the largest deletion defined by molecular analysis (D14S53 to D14S67). The physical size of band $14 \mathrm{q} 31$ can be estimated to be $8 \cdot 8 \mathrm{Mb}^{2324}$ Patient 2 , initially described as having a terminal deletion of chromosome 14, in reality has a smaller deletion of band $14 \mathrm{q} 31$ which overlaps with that of patient 1 (D14S74 to D14S67). The phenotypes of the two patients also overlap, although the abnormalities are non-specific; both have epicanthic folds, dental abnormalities, protuberant ears, narrowed palate, delayed speech, and mild developmental delay.

Patient 3 and his mother were defined by karyotype analysis as having an interstitial deletion of part of band 14q31 inherited from the maternal grandfather. They have the smallest deletion detected by molecular analysis, consisting of a single anonymous DNA marker, D14S73. Patient 3 also has the most normal phenotype, characterised by cupped ears and mild developmental and speech delay. It is difficult to estimate the amount of DNA deleted in the absence of a physical map of this region. However, the genetic distance between D14S48 and D14S81 has been estimated on the CEPH Consortium linkage map to be $10 \mathrm{cM}$, so the physical distance may also be small. The deletion in patient 3 lies within that of patient 1 , and presumably within that of patient 2, who is uninformative at D14S73. The order of markers D14S68, D14S73, and D14S67 could not be confirmed by these deletions. The very small deletion seen in patient 3 and his mother is consistent with the mild phenotype; a number of small deletions or duplications of chromosome bands have been seen in phenotypically normal subjects. ${ }^{25}$

These results localise a number of genetic markers to the chromosome band $14 \mathrm{q} 31$, providing a link between the genetic and physical maps of chromosome 14. The order of markers derived from the deletions is consistent with the linkage maps ${ }^{1116}$ and the data from analysis of somatic cell hybrids. ${ }^{17}$ Deletions in patients 1 and 2, but not likely in patient 3, may overlap with the region to which the gene for MachadoJoseph disease has been localised by linkage analysis, just proximal to D14S81 (fig 4). ${ }^{78}$ Following the initial submission of this report, the MJD gene was cloned and found to contain an unstable repeat in the coding region. At present, it is not known whether MJD results from loss or gain of function. If the former is true, patients 1 and 2 may be at risk for the development of MJD and further analysis is required.
We are grateful to Drs Philip E Podruch and Fu-sun Yen, University of Louisville, for patient samples, to Gail Billingsley and Van Nguyen for technical assistance, to Diana de Vos for assistance in preparing the flow sorted chromosomes from
patient 1 , to Gloria Nie for the karyotype studies of patient 3 patient 1 , to Gloria Nie for the karyotype studies of patient 3
and his family, and to Gail Billingley and Richard Wintle for critical reading of the manuscript. This work was supported by a grant (MT 5426) from the Medical Research Council of a grant (MT 5426)

1 Gorski JL, Uhlmann WR, Glover TW. A child with multiple congenital anomalies and karyotype 46,XY,del(14) (q31q32.3): further delineation of chromosome 14 interstitial deletion syndrome. Am f Hum Genet 1990;37: terstitia

2 Karnitis SA, Burns K, Sudduth KW, Golden WL, Wilson WG. Deletion (14) (q24.3q32.1): evidence for a distinct WG. Deletion (14) (q24.3q32.1): evidence for a disting
clinical phenotype. Am $\mathfrak{F}$ Med Genet 1992;44:153-7.

3 Rivera H, Ramirez-Duenas ML, Figuera LE, GonzalezMontes RM, Vasques AI. Opposite imbalances of dista $14 \mathrm{q}$ in two unrelated patients. Ann Genet (Paris) 1992 35:97-100.

4 Yen FS, Podruch PE, Weisskopf B. A terminal deletion (14) (q31.1) in a child with microcephaly, narrow palate, gingival hypertrophy, protuberant ears and mild mental retardation. $\mathcal{F}$ Med Genet 1989;26:130-3.

5 Wintle RF, Costa T, Haslam RHA, Teshima IE, Cox DW. Molecular analysis redefines three human chromosome 14 deletions. Hum Genet (in press)

6 Takiyama $Y$, Nishizawa $M$, Tanaka $H$, et al. The gene for Machado-Joseph disease maps to human chromosome 14. Nature Genet 1993;4:300-3.

7 Sasaki H, Wakisaka A, Takada A, et al. Mapping of the gene for Machado-Joseph disease within a 3.6-cM interval flanked by D14S291/D14S280 and D14S81, on the basis of studies of linkage and linkage disequilibrium in 24 of studies of linkage and linkage disequilibrium in

8 St George-Hyslop P, Rogaeva E, Hutterer J, et al. MachadoJoseph disease in pedigrees of Azorean descent is linked to chromosome 14. Am f Hum Genet 1994;55:120-5.

9 Kawaguchi Y, Okamoto T, Taniwaki M, et al. CAG expansions in a novel gene for Machado-Joseph disease at chromosome 14q32.1. Nature Genet 1994;8:221-7.

10 Sharma V, Smith L, Allen L, Magenis RE, Litt M. Dinucleotide repeat polymorphisms at the D14S43 locus. Nucleic Acids Res 1991;19:1722.

11 Weissenbach J, Gyapay G, Dib C, Vignal A, Morisette J, Millasseau P. A second generation linkage map of the human genome. Nature 1992;359:794-9.

12 Wang $Z$, Weber JL. Continuous linkage map of human chromosome 14 short tandem repeat polymorphisms. Gen omics 1992;13:532-6.

13 Gerken S. Data deposited in the Genome Data Base (GDB) 1993.

14 Byth BC, Cox DW. A (CA) nepeat at the $5^{\prime}$ end of the $\alpha_{1}$ antitrypsin gene. Hum Mol Genet 1993;2:1752.

15 Byth BC, Cox DW. Two consecutive dinucleotide repeats constitute an informative marker at the $\alpha_{1}$-anticonstitute an informative marker at the $\alpha_{1}$-anti1085 .

16 Cox DW, Billingley GD, Nguyen VT. A linkage map of human chromosome 14, including 13 gene loci. Genomics 1994;23:331-7.

17 Billingsley GD, Cox DW, Duncan AMV, Goodfellow PJ Grzeschik K-H. Regional localization of loci on chromosome 14 using somatic cell hybrids. Cytogenet Cell Gene 1994;66:33-8.

18 Cox DW, Gedde-Dahl T, Menon AG, et al. Report of the second international workshop of human chromosome 14 mapping, 1994. Cytogenet Cell Genet 1995;69:160-74.

19 Béroud C, Fournet JC, Jeanpierre C, et al. Microsatellite mapping defines a $3 \mathrm{Mbp}$ region in $14 \mathrm{q} 24.3$ commonly deleted in renal cell carcinoma. (Submitted.)

20 Carter NP, Ferguson-Smith ME, Affara NA, Briggs $H$, Ferguson-Smith MA. Study of $\mathrm{X}$ chromosome $\mathrm{ab}-$ normality in XX males using bivariate flow karyotype analysis and flow sorted dot blots. Cytometry 1990;11: analysis

21 Rabin M, Watson M, Kidd V, Woo SLC, Breg WR, Ruddle FH. Regional location of $\alpha_{1}$-antichymotrypsin and $\alpha_{1}$ antitrypsin genes on human chromosome 14. Som $\mathrm{Ce}$ Mol Genet 1986;12:1209-14.

22 Billingley GD, Nguyen VT, Cox DW. Physical and genetic localization of loci in the mid region of chromosome 14q Cytogenet Cell Genet 1991;58:1986.

23 Daniel A. The size of prometaphase chromosome segments. Clin Genet 1985;28:216-24.

24 Morton NE. Parameters of the human genome. Proc Nat Acad Sci USA 1991;88:7474-6.

25 Bortotto L, Piovan E, Furlan R, Rivera H, Zuffardi O Chromosome imbalance, normal phenotype, and im printing. $\mathcal{F}$ Med Genet 1990;27:582-7. 